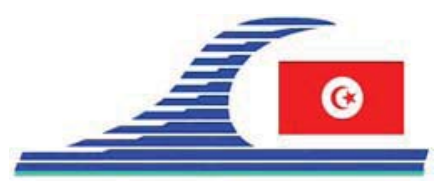

\author{
Conférence Méditerranéenne Côtière et Maritime \\ EDITION 1, HAMMAMET, TUNISIE (2009) \\ Coastal and Maritime Mediterranean Conference \\ Disponible en ligne - http://www.paralia.fr-Available online
}

\title{
Caractérisation des tempêtes et de leur évolution sur le littoral du Golfe du Lion
}

\author{
Yann BALOUIN ${ }^{1}$, Mathieu GERVAIS ${ }^{1}$, Rémi BELON ${ }^{1}$, \\ Gonéri LE COZANNET ${ }^{2}$, Sophie LECACHEUX ${ }^{2}$, Yann KRIEN ${ }^{2}$, \\ Déborah IDIER ${ }^{2}$, Rodrigo PEDREROS ${ }^{2}$
}

1. BRGM, Service Géologique du Languedoc-Roussillon, 1039, rue de Pinville, 34000 Montpellier, France.

y.balouin@brgm.fr

2. BRGM, RNSC-RIC, 3 Av; Claude Guillemin, B.P.36009, 45060 Orléans, France.

\section{Résumé :}

Dans le cadre du projet européen MICORE, une analyse des événements historiques de tempêtes ayant affecté le littoral du Golfe du Lion a été réalisée. Les caractéristiques physiques des tempêtes marines (hydrodynamique, météorologie), ainsi que les réponses morphologiques et les impacts socio-économiques induits ont été analysés pour déterminer les seuils morphogènes des tempêtes et pour évaluer les évolutions éventuelles de la fréquence et de l'intensité de ces événements au cours des dernières décennies. Les résultats obtenus mettent en évidence une grande variabilité interannuelle des tempêtes et l'absence de tendance clairement établie. La durée des tempêtes, la hauteur des vagues et des surcotes, et la fréquence des événements ne présentent aucune tendance significative au cours de la période étudiée.

\section{Mots-clés :}

Tempêtes - Seuils morphogènes - Impacts morphologiques - Changement climatique

\section{Introduction}

Les tempêtes entraînent souvent des réponses morphologiques rapides et importantes sur les littoraux sableux: érosion du trait de côte, submersion de la plage, franchissements dunaires, mais également une importante dynamique des barres d'avant-côte. Le projet européen MICORE (Morphological Impacts and Coastal Risk induced by Extreme storm events), qui associe 16 partenaires de 9 pays, a pour objectif principal de développer un démonstrateur d'outils intégrés pour la prévision des impacts morphologiques des tempêtes en soutien aux stratégies de protection civile. Le projet vise à contribuer au développement d'une cartographie probabiliste des impacts morphologiques et à la production d'un système d'alerte et d'information pour la réduction à long terme des dommages. La région française concernée est le Golfe du Lion où une analyse historique a été réalisée pour caractériser les événements passés et mettre en évidence d'éventuelles évolutions de leur fréquence et de leur intensité.

DOI: $10.5150 / \mathrm{cmcm} .2009 .020-2$ 


\section{Données disponibles et analyses}

Afin de caractériser les tempêtes affectant le Golfe du Lion, les données disponibles ont été utilisées. Les mesures sont relativement récentes dans le Golfe du lion. Il s'agit principalement des données de vagues non directionnelles (bouées, 1989-2009) ainsi que des données des marégraphes (1986-2000) du SMNLR puis de la DRE-LR. Afin d'obtenir des séries temporelles de vagues plus longues, les résultats des simulations effectuées lors du projet HIPOCAS ont été ré-analysées. Elles permettent d'obtenir des informations sur la houle de 1958 à 2001. La validité de ces simulations a été analysée par comparaisons avec les séries mesurées. Afin d'améliorer les résultats, les données de vent NCEP (1979-2009) ont également été utilisées pour générer des séries temporelles de houle à l'aide du modèle de propagation SWAN (BOOIJ et al., 1999).

Par ailleurs, des données morphologiques provenant de la DRE-LR ont été utilisées et une importante recherche des observations d'impacts a été réalisée afin d'évaluer les seuils morphogènes. 55 événements ayant entrainé des impacts morphologiques et/ou socio-économiques ont ainsi été recensés.

\section{Caractérisation des tempêtes dans le Golfe du Lion et tendance d'évolution}

Différents indicateurs tels que la création de brèches, les franchissements dunaires, le recul du trait de côte, les volumes érodés, l'extension de la submersion, la surcote, la dynamique des barres d'avant-côte, ont permis de caractériser les seuils morphogènes. Il découle de cette analyse que : (1) les évolutions morphologiques majeures surviennent dès lors que la hauteur significative de la houle au large atteint $4 \mathrm{~m}$, (2) des impacts morphologiques importants (franchissement $\mathrm{du}$ cordon, dommages sur les infrastructures) sont observés lorsque la hauteur significative atteint $5 \mathrm{~m}$. Ces seuils morphogènes sont clairement inférieurs si plusieurs tempêtes d'occurrence annuelle surviennent lors du même hiver.

Les impacts socio-économiques ont également été analysés. Ces impacts concernent principalement les dommages sur les défenses côtières et les infrastructures de front de mer (routes, restaurants, ports). Cette approche s'est avérée difficile car l'obtention de données exhaustive est quasi-impossible. Par ailleurs, la perception de l'impact par les populations influe fortement sur les dommages recensés. De plus, un événement majeur est généralement suivi de la mise en place de défenses côtières plus lourdes, ce qui rend difficile la confrontation des impacts avec l'énergie déployée lors de la tempête. Les impacts socio-économiques recensés et les coûts induits augmentent généralement avec l'intensité des tempêtes, mais ils dépendent fortement du degré d'anthropisation du littoral et de l'historique du secteur.

\section{Evolution de la fréquence et de l'intensité des tempêtes}

En utilisant les données et simulations disponibles, l'intensité et la fréquence des tempêtes a été analysée pour déterminer d'éventuelles tendances sur les 50 dernières 
années. Les simulations SIMAR des états de mer passés ont été corrigées et validées à l'aide des données mesurées. Les critères analysés sont : le nombre d'événements par an et par hiver, les hauteurs de vagues maximales, les moyennes annuelles de ces hauteurs, la durée des événements, l'élévation maximale et moyenne des surcotes, ...

Les données de vagues mesurées couvrant une très faible période ne permettent pas de dégager des tendance d'évolution, comme cela a déjà été constaté par plusieurs auteurs (ULLMAN, 2008 ; SABATIER et al., 2009). Elles demeurent toutefois utiles pour calibrer et valider les séries simulées. Ces données mettent en évidence une légère augmentation (peu représentative statistiquement) de la fréquence des événements dans les années 90. Les mesures du marégraphe de Sète ne permettent pas de caractériser d'évolution de la surcote. La surcote moyenne à Sète est de l'ordre de $35 \mathrm{~cm}$, et les surcotes maximales annuelles fluctuent fortement entre 40 et $80 \mathrm{~cm}$. L'augmentation du niveau moyen mesuré par ce marégraphe est de l'ordre de $2.9 \mathrm{~mm} / \mathrm{an}$, ce qui est en accord avec les observations satellitales (CAZENAVE et al., 2002).

Les simulations HIPOCAS ont donc été ré-analysées pour appréhender l'évolution sur une période plus importante. Une classification de ces données de houle a été réalisée avec un algorithme de K-moyennes pour étudier spécifiquement les classes les plus énergétiques (figure 1). La hauteur de vagues présente une grande variabilité et aucune tendance significative n'est observée. La fréquence des événements des classes énergétiques (Swell 1 et 2, Storm) présente une augmentation (peu significative) depuis les années 1970, qui correspond à $+0.3 \mathrm{j} /$ an (cf. figure 2). La variabilité interannuelle reste toutefois importante, et l'occurrence des tempêtes pour les périodes (1958-1970) et (2004-2008) tend à diminuer. De plus, la fréquence de la classe la plus énergétique (Storm) est stable sur ces 40 ans.

\section{Conclusions}

Dans le cadre du projet MICORE, les données hydrodynamiques, météorologiques, morphologiques et socio-économiques disponibles ont été analysées pour caractériser les seuils morphogènes des tempêtes affectant le Golfe du Lion. Des seuils morphogènes ont ainsi pu être évalués à partir de données quantitatives (hauteurs de vagues, évolutions morphologiques, ...) et qualitatives (dommages, ...). Les seuils de tempêtes ayant un impact morphologique ou socio-économique important correspondent à des tempêtes d'occurrence annuelles à biannuelles.

Différents indicateurs ont été analysés pour évaluer les possibles tendances d'évolution des tempêtes au cours des dernières décennies. Les analyses des mesures à court-terme et des simulations à moyen terme mettent en évidence une relative stabilité de la fréquence et de l'intensité des tempêtes. Des tendances à l'augmentation de la fréquence des événements des années 1970 à 2000 sont observées. Toutefois, elles sont peu significatives étant donnée la forte variabilité interannuelle des événements. En 
conséquence, aucune relation n'a pu être caractérisée entre l'évolution des tempêtes et le changement climatique global.

Malgré l'absence de tendance dans ces analyses, les résultats obtenus n'impliquent pas que des tendances à plus long-terme n'existent pas, ni que les changements climatiques actuels et futurs n'auront pas d'effets sur les régimes de tempêtes dans le futur.

\begin{tabular}{|l|c|c|c|c|}
\hline Description & Hm0 & Tp & Dm & Occurrence \\
\hline Tramontane, N, annual & 0.29 & 2.38 & 7 & $13.2 \%$ \\
\hline Tramontane, NO, annual & 0.47 & 2.56 & 315 & $14.8 \%$ \\
\hline Tramontane, NO, annual & 0.88 & 3.26 & 347 & $9.5 \%$ \\
\hline Tramontane, NO, annual & 0.93 & 3.22 & 310 & $6.7 \%$ \\
\hline INTER, S, annual & 0.34 & 5.91 & 167 & $7.8 \%$ \\
\hline INTER, SE, annual & 0.28 & 3.70 & 159 & $20.1 \%$ \\
\hline INTER, SE, annual & 0.72 & 4.14 & 150 & $13.0 \%$ \\
\hline INTER, SE, annual & 1.00 & 5.81 & 136 & $6.3 \%$ \\
\hline SWELL1, E, automn, winter, spring & 0.84 & 9.20 & 96 & $1.7 \%$ \\
\hline SWELL2, S, automn, winter, spring & 1.27 & 7.16 & 171 & $3.2 \%$ \\
\hline SWELL2, SE, automn, winter, spring & 1.80 & 7.25 & 133 & $2.8 \%$ \\
\hline STORM SE, november to march & 3.13 & 9.00 & 124 & $0.7 \%$ \\
\hline
\end{tabular}

Figure 1. Classification de la houle au large de Sète (simulations HIPOCAS corrigées).

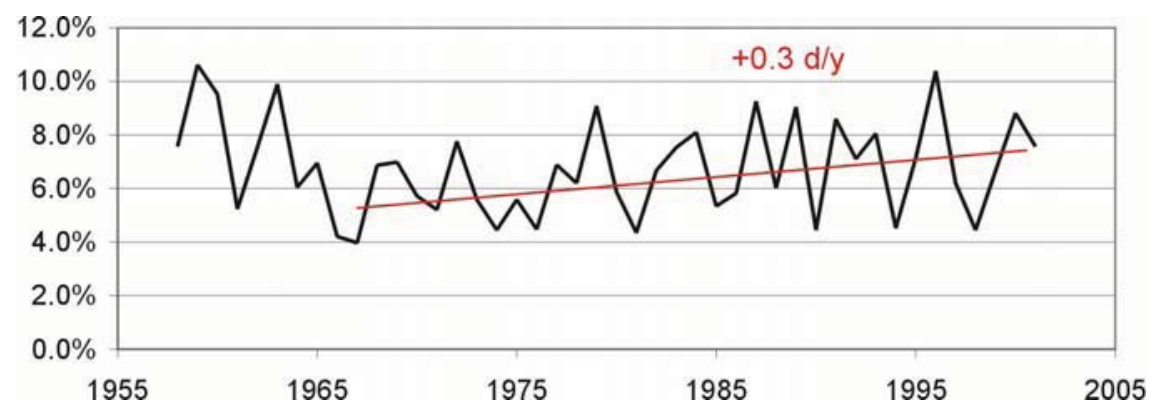

Figure 2. Fréquences cumulées des classes les plus énergétiques (Swell 1 et 2, Storm).

\section{Références bibliographiques}

BOOIJ N., RIS R.C., HOLTHUIJSEN L.H. (1999). A third-generation wave model for coastal regions, Part I, Model description and validation. J.Geoph.Research C4, 104, pp 7649-7666.

CAZENAVE A., BONNEFOND P., MERCIER F., DOMINH K., TOMAZOU V. (2002). Sea level variations in the Mediterrannean Sea and Black Sea from satellite altimetry. Global and Planetary Change, 34, pp 59-86.

SABATIER F., SAMAT O., ULLMANN A., SUANEZ S. (2009). Connecting largescale coastal behaviour with coastal management of the Rhône delta. Geomorphology, 107, pp 79-89.

ULLMANN A. (2008). Surcotes dans le Golfe du Lion et conditions atmosphériques: variabilité contemporaine et future (1905-2100). Thèse, University of Aix-Marseille I, $236 \mathrm{p}$. 